\title{
Ökonomische Aspekte des Klimaschutzes
}

\author{
Stefan Bayer*
}

\begin{abstract}
The paper concentrates on some economic aspects of climate change. The fundamental trends and the scientific state of the art are presented firstly, followed by some basic economic implications. After that our considerations focus on two important economic aspects of climate change: Chapter two highlights its long-term character and the asymmetric distribution of benefits and costs, leading to a political bias in favor of short-term political action. Chapter three describes and investigates the tradable permit approach to reach politically given climate targets. Our analysis stresses the advantages of this instrument and offers reasons why its political configuration must be seen as main reason for some deficiencies in reducing GHG-emissions.
\end{abstract}

Keywords: Klimarahmenkonvention, Kyoto-Protokoll, Langfristphänomen, Diskontierung, Globalschadstoff, Handelbare Emissionsrechte

\section{Einleitung}

$\mathrm{D}$ ie Konzentration von Treibhausgasen in der Erdatmosphäre hat im Vergleich zum vorindustriellen $\mathrm{Ni}$ veau Mitte des 18. Jahrhunderts stark zugenommen. Dies hat zur Folge, dass ein Teil der Wärmestrahlung von der Erde nicht mehr ins Weltall abgegeben werden kann, sondern - wie in einem Treibhaus - auf die Erde zurückgeworfen wird, und es zu einer durchschnittlichen Erwärmung der Erdoberfläche kommt. ${ }^{1}$ Die Wissenschaft spricht in diesem Fall vom anthropogenen Treibhauseffekt, der die Strahlungsbilanz der Erde verändert. Weil einerseits die Wärmestrahlung von der Spurengasschicht reflektiert wird und andererseits die Sonnenstrahlung ungehindert diese Schicht passiert, erhöht sich die Nettozufuhr von Wärme durch die Sonne auf der Erde. Vom anthropogenen Treibhauseffekt muss der natürliche Treibhauseffekt unterschieden werden, ohne den die Durchschnittstemperatur auf der Erde in Bodennähe bei etwa $-19^{\circ} \mathrm{C}$ liegen würde. Erst der natürliche Treibhauseffekt ermöglicht der Menschheit ein Leben, wie wir es kennen. Die Emissionen von Treibhausgasen verstärken den natürlichen Treibhauseffekt, so dass die Durchschnittstemperatur auf der Erdoberfläche ansteigt. Dieser vom Menschen verursachte Effekt („Klimawandel“) gilt spätestens seit dem 4. Sachstandsbericht des IPCC aus dem Jahre 2007 als Stand der Wissenschaft, auch wenn ihn verschiedentlich Klimaskeptiker anzweifeln. ${ }^{2}$

Das bedeutendste Treibhausgas ist Kohlendioxid, das in erster Linie bei der Verbrennung fossiler Brennstoffe wie Kohle, Öl und Gas anfällt. Weitere wichtige Treibhausgase sind Methan, Distickstoffoxid, Fluorchlorkohlenwasserstoffe und troposphärisches Ozon. Alle Treibhausgase wirken wie ein „Globalschadstoff", d.h. sie entfalten ihre spezifische Treibhauswirkung (Treibhauspotenzial) unabhängig vom Emissionsort. Die Emittenten von Treibhausgasen verteilen sich über die gesamte Erde und sind in nahezu allen volkswirtschaftlichen Sektoren zu finden. Wirksamer Klimaschutz muss deshalb international

\footnotetext{
* Priv.-Doz. Dr. Stefan Bayer, Dozent für Ökonomie/Ökologie am Fachbereich Sozialwissenschaften der Führungsakademie der Bundeswehr und Privatdozent für Volkswirtschaftslehre an der Eberhard-Karls-Universität Tübingen. Der Autor dankt zwei anonymen Gutachtern für ihre konstruktiven Kommentare.

1 Vgl. IPCC (2007a).

2 Vgl. exponiert etwa Lomborg (2007), der allerdings vornehmlich aus statistischer Perspektive argumentiert.
}

betrieben werden. Gleichwohl verursachten die Industrieländer historisch einen Großteil der weltweiten treibhausrelevanten Emissionen und sie werden auch auf absehbare Zeit den Löwenanteil dieser Emissionen ausstoßen. Hinzu addieren sich mittlerweile nennenswerte Treibhausgasemissionen auch einiger Entwicklungsländer, zu denen nach internationaler Terminologie v.a. China und Indien zählen. Alle Treibhausgase weisen zudem unterschiedliche meist sehr lange Verweildauern in der Atmosphäre auf (z. B. $\mathrm{CO}_{2} 50$ bis 200 Jahre), was zu Konflikten zwischen Kurzzeit- und Langzeitinteressen führen kann.

Der nachfolgende Beitrag soll anhand zweier Facetten wirtschaftliche Einflüsse beim Klimawandel und Klimaschutz analysieren und Implikationen erörtern. Zunächst soll in Kapitel zwei die Langfriststruktur des Klimawandels in den Mittelpunkt gerückt und die damit verbundenen Implikationen im Rahmen einer ökonomischen Analyse offen gelegt werden. Daran anschließend wird in Kapitel drei die grundsätzliche Vorzugswürdigkeit eines handelbaren Emissionsrechtesystems bei der Bekämpfung des Klimawandels herausgearbeitet, um dieses System in einem zweiten Schritt unter Berücksichtigung mannigfacher ökonomischer Interessen in seiner praktischen Ausgestaltung genauer zu untersuchen. Das Fazit in Kapitel vier beschließt unsere ökonomischen Überlegungen.

\section{Der Klimawandel als Langfristphänomen und dessen ökonomische Analyse}

Neben der regionalen Divergenz von Ursachen und Wirkungen, die in Kapitel drei in den Mittelpunkt rückt, fallen Emissionen und daraus resultierende Klimaschäden auch zeitlich auseinander. Heute emittierte Treibhausgase entfalten ihre spezifische Treibhauswirksamkeit nicht nur zum jetzigen Zeitpunkt, sondern bleiben unterschiedlich lange in der Atmosphäre wirksam, einzelne Gase gar bis zu mehreren tausend Jahren. Dies führt zu Konflikten zwischen Kurzzeit- und Langzeitinteressen. Heutige Emissionen verursachen möglicherweise erst in 100 Jahren Schäden, etwa in Form des Anstiegs des Meeresspiegels und der Überschwemmung küstennaher Regionen. Auf der anderen Seite führen heute vermiedene Emissionen erst in etwa 30 bis 40 Jahren zu spürbaren Klimaverbesserungen, ab diesem Zeitpunkt jedoch möglicherweise bis in die weite Zukunft. Die 
langen Zeitverzögerungen liegen vor allem an den erheblichen Trägheiten des weltweiten Klimasystems. Insofern erfordert die Bekämpfung des anthropogenen Klimawandels einen umfassenden Ansatz: Wirksamer Klimaschutz muss sowohl international als auch unter Berücksichtigung der intertemporalen Auswirkungen, also dauerhaft, betrieben werden.

Ökonomische Überlegungen zum Klimawandel finden im Rahmen monetärer Vorteilhaftigkeitskalküle statt, ${ }^{3}$ d.h., es wird eine Geldrechnung angestellt. Mit der Bewertung in Geld soll erreicht werden, dass die Vor- und Nachteile des Klimaschutzes (bzw. des Klimawandels als analoge Frage) in vergleichbarer Weise erfasst werden; Kosten von Klimaschutzmaßnahmen werden also mit den daraus resultierenden Nutzen kontrastiert. Die ökonomische Forschung beschäftigt sich deshalb intensiv mit der monetären Bewertung von Effekten, für die keine Marktpreise vorliegen. Insbesondere in der umweltökonomischen Bewertungsforschung wurden in den letzten Jahren deutliche Fortschritte gemacht, die mittlerweile auch Einzug in die politische Debattenlage gehalten haben. ${ }^{4}$ Wir wollen den Bewertungsaspekt im Rahmen dieses Aufsatzes allerdings nicht weiter thematisieren, sondern stattdessen auf die Notwendigkeit abstellen, die zu unterschiedlichen Zeitpunkten auftretenden monetären Effekte miteinander zu vergleichen. Der Betrag von einem Euro ist zu unterschiedlichen Zeitpunkten unterschiedlich viel wert. Er kann etwa angelegt werden und wäre - bei einem angenommenen Zinssatz von drei Prozent p.a. - in einem Kalenderjahr 1,03 Euro wert. Umgekehrt könnte auch gefragt werden, wie viel Geld man heute sparen müsste, um beim gleichen fingierten Zinssatz in einem Jahr einen Betrag von einem Euro zu erhalten: Diese Rechnung führt zu einem Betrag von (gerundeten) 0,97 Euro. Wenn Ökonomen also eine Geldrechnung anstellen, muss die Analyse berücksichtigen, dass Geld zu unterschiedlichen Zeitpunkten unterschiedlich viel wert ist; die ökonomische Analyse benötigt somit für Langfristüberlegungen ein Verfahren, um zu unterschiedlichen Zeitpunkten auftretende Effekte miteinander vergleichbar machen zu können. Die Geldgrößen müssen aus Gründen der Vergleichbarkeit auf einen einheitlichen Zeitpunkt, typischerweise den Zeitpunkt der Planung, bezogen werden. Dies geschieht durch Diskontierung. Die Berücksichtigung des intertemporalen Aspekts macht ein dynamisches Planungsverfahren erforderlich. Man verwendet im Allgemeinen die Kapitalwertmethode. Der Kapitalwert einer Investition entspricht dem Barwert der erwarteten Nettoerträge (Erträge minus Kosten) abzüglich des Barwerts der Investitionsausgaben. Eine Investition ist vorteilhaft, wenn der Kapitalwert größer oder gleich null ist. ${ }^{5}$

Als wichtigste Diskontierungsgründe - neben der hier nicht betrachteten Unsicherheit - sind die folgenden Faktoren zu nennen: ${ }^{6}$

1. Gegenwärtige Bedürfnisse werden von den Individuen grundsätzlich höher bewertet als zukünftige (reine Zeitpräfe-

3 Vgl. Cansier/Bayer (2003), S. 217-240.

4 Eine der derzeit prominentesten Studien ist die des britischen Ökonomen Sir Nicholas Stern (2006), der die ökonomischen Folgen, also die monetären Nutzen und Kosten, des Klimawandels prognostiziert. Einführend zu diesem Themenkomplex sei auf Cansier/Bayer (2003), Kapitel XI, S. 217ff. hingewiesen.

5 Vgl. Cansier/Bayer (2003), S. 217f. und S. $230 f$.

6 Vgl. zur weiteren Vertiefung Bayer (2000), S. 25-41. renz). Man verfügt lieber heute über ein bestimmtes Bündel von Konsumgütern als irgendwann in der Zukunft. Die Menschen haben eine Präferenz für die Gegenwart. Als Hauptgründe hierfür werden die menschliche Ungeduld und Kurzsichtigkeit (Myopie) genannt.

2. Menschen erwarten, dass sie später reicher sein werden und bewerten deshalb einen gegebenen zusätzlichen Konsum in der Zukunft geringer als in der Gegenwart (wachstumsbedingte Zeitpräferenz). Voraussetzungen sind hierbei ein steigendes ProKopf-Einkommen und Gültigkeit der Hypothese vom fallenden Grenznutzen des Konsums mit steigendem Einkommen. Diese Zeitpräferenzrate bestimmt sich nach der Wachstumsrate des Pro-Kopf-Einkommens und dem Ausmaß der Verringerung des Grenznutzens mit steigendem Konsum (sog. Elastizität des Grenznutzens des Konsums). ${ }^{7}$

3. Wenn der Staat einen bestimmten Eurobetrag für ein öffentliches Projekt einsetzt, dann werden unter Umständen in diesem Umfang private Investitionen verdrängt. Diese Investitionen wären mit einer bestimmten privaten Ertragsrate verbunden gewesen. Auf diese Ertragsrate wird verzichtet. Die so entgangenen Erträge stellen die Opportunitätskosten des öffentlichen Projekts dar. Deshalb wird verschiedentlich auch vorgeschlagen, die private Ertragsrate von Investitionen als Diskontierungsrate bei öffentlichen Vorhaben zu verwenden.

Die nachfolgende Graphik soll einen Eindruck vermitteln, welche Auswirkungen die Diskontierung mit einer (im internationalen Rahmen relativ niedrigen) Rate von drei Prozent auf einen Effekt in Höhe von $1.000 €$ hat, der zu unterschiedlichen Zeitpunkten in der Zukunft auftritt.

Gehen wir davon aus, dass der Klimawandel in 90 Jahren einen Schaden in Höhe von $1.000 €$ induziert. Dieser Schaden ist aus heutiger Sicht nur 69,90 Euro wert, träte er in 150 Jahren auf, betrüge sein heutiger Wert (Barwert) nur noch 11,90 Euro etc. Bei höheren Diskontierungsraten beschleunigt sich der Abwertungsprozess, bei niedrigeren verzögert er sich dagegen. Grundsätzlich bewirkt die Diskontierung mit positiven Raten, dass zeitlich entfernt auftretende Effekte mit deutlich geringerem Gewicht in heute anstehende Entscheidungsprozesse eingehen als zeitnäher auftretende. Langfristige Auswirkungen finden in heutigen Entscheidungen allenfalls geringe Berücksichtigung. Dies ist sowohl ineffizient als auch ungerecht, da bestimmte Kosten nicht bei ihren Verursachern zur Anrechnung kommen, wodurch diese sich im Zeitverlauf einen Vorteil verschaffen. Tendenziell führt dies dazu, dass

1. Vorhaben, bei denen zeitnahe Kosten zukünftigen Nutzen gegenüberstehen, nicht realisiert werden (etwa Klimaschutzmaßnahmen) und

2. Vorhaben, bei denen zeitnahe Nutzen zukünftigen Kosten gegenüberstehen, realisiert werden (etwa Nutzung von Atomstrom als kostengünstige Alternative zu fossil erzeugtem Strom).

Dabei gilt, dass die Asymmetrien mit steigenden Diskontierungsraten stärker ins Gewicht fallen. Umgekehrt gilt auch, dass geringere Diskontierungsraten weniger starke Asymmetrien verursachen.

\footnotetext{
7 Vgl. ausführlicher Bayer (2000), S. 107-159.
} 
Abbildung 1: Barwert eines Effektes von tausend $€$ in 1 bis 300 Jahren bei einer Diskontierungsrate von 3 Prozent p.a., eigene Darstellung.

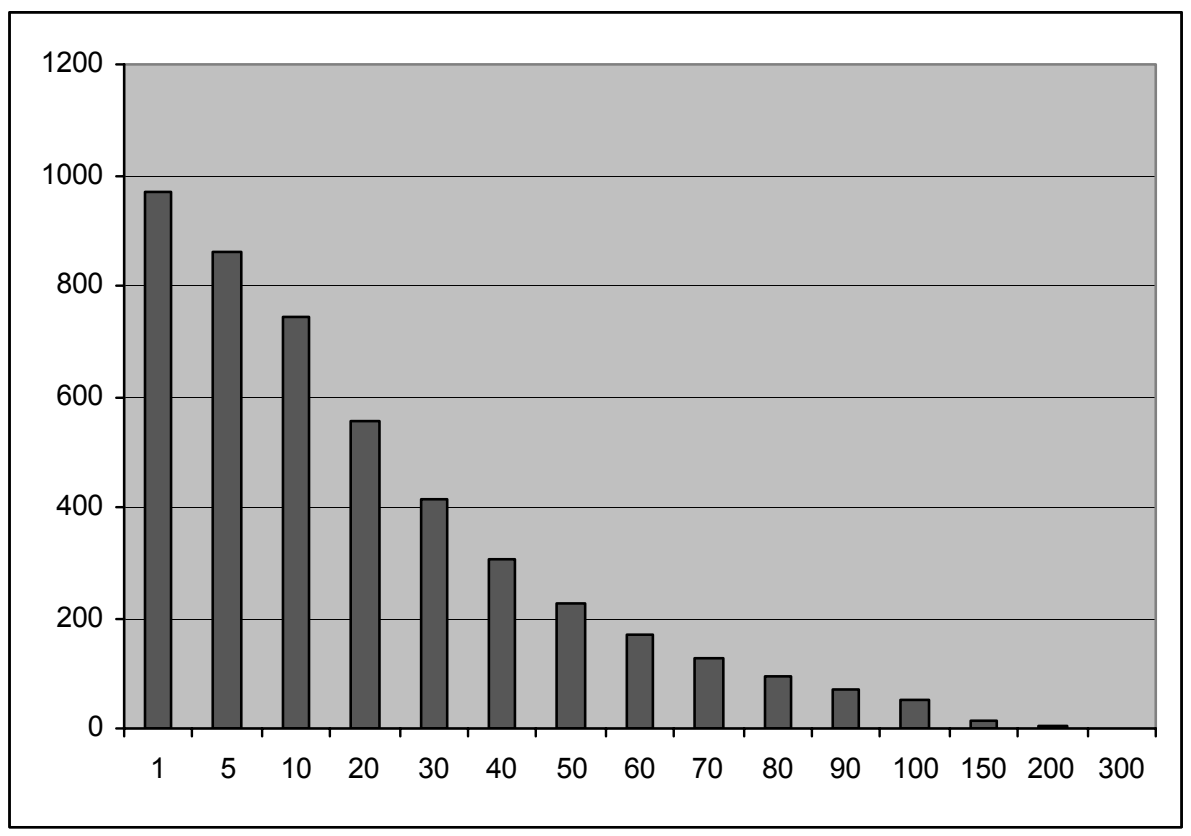

Vor dem Stern-Report aus dem Jahr 2006 hat William Nordhaus das prominenteste und auch politisch einflussreichste Beispiel einer ökonomischen Analyse des Klimawandels vorgelegt und damit maßgeblich auch Präsidenten der USA beraten. ${ }^{8} \mathrm{Er}$ nimmt eine Bilanzierung der Kosten und Nutzen für die USA bis Mitte des übernächsten Jahrhunderts vor. Die Diskontierungsrate nimmt im Zeitverlauf von anfänglich sechs Prozent auf drei Prozent ab. Für die Klimaschadensschätzung wird angenommen, dass eine Verdoppelung der $\mathrm{CO}_{2}$-Konzentration in der Atmosphäre gegenüber dem vorindustriellen Niveau bis zum Jahr 2100 mit einer Verringerung des Weltsozialproduktes um 1,33 Prozent verbunden ist. Für die Kosten der Vermeidung von Treibhausgasemissionen wird angenommen, dass die Reduktion der laufenden $\mathrm{CO}_{2}$-Emissionen um 50 Prozent gegenüber der unkontrollierten Entwicklung Kosten in Höhe von einem Prozent des Weltsozialproduktes der jeweiligen Periode aufwirft. Auf dieser Basis leitet Nordhaus einen Optimalpfad ab, der nahezu identisch mit der Entwicklung bei Politikverzicht ist, für die die Naturwissenschaftler mit hoher Wahrscheinlichkeit eine Klimakatastrophe prognostizieren (Erhöhung der globalen Temperatur um $3,2^{\circ} \mathrm{C}$ statt um $3,4^{\circ} \mathrm{C}$ bis zum Ende des nächsten Jahrhunderts). Kurz zusammengefasst kann man festhalten: Klimaschutz verursacht aus Sicht der USA zu hohe Kosten, ohne dass diesen ausreichend Erträge gegenüberstehen. Die Investition in Klimaschutz wäre somit ineffizient.

Kritisiert werden müssen allerdings einige Annahmen des Modells von Nordhaus: So verwendet er eine sehr hohe Diskontierungsrate (6 Prozent zu Beginn, 3 Prozent am Ende der Berechnungsperiode), die dazu führt, dass zukünftige Klimaschäden aus heutiger Sicht deutlich weniger ins Gewicht fallen als bei

8 Vgl. Nordhaus (1994). geringeren Raten. Bayer zeigt etwa ${ }^{9}$, dass das Modell von Nordhaus bei Verwendung niedrigerer Diskontierungsraten von etwa einem Prozent oder gar null Prozent zu deutlich höheren optimalen Vermeidungsleistungen rät. Insbesondere unter Berücksichtigung der ökonomischen Notwendigkeit einer Vollkostenrechnung stellt sich die berechtigte Frage, ob nicht die Kostenanlastung sowohl im Ausland als auch in der Zukunft der USA heute einen Wettbewerbsvorsprung sichert, indem bei Dritten klimainduzierte Schäden verursacht werden. Auch der bereits erwähnte Stern-Report ${ }^{10}$ verwendet eine deutlich geringere Diskontierungsrate als etwa Nordhaus und kommt nicht zuletzt deswegen zu seiner zentralen Empfehlung, dass heutige Klimaschutzpolitik gegenüber seiner Unterlassungsalternative ökonomisch vorzugswürdig wäre. Diese Ausführungen verdeutlichen die strategische Bedeutung der Höhe der Diskontierungsrate. Bereits geringe Variationen ihres Niveaus führen bei sonst gleichen Bedingungen und Modellspezifikationen zu gänzlich anderen Ergebnissen, wie am Beispiel des NordhausModells angedeutet wurde.

Darüber hinaus rechnen sehr hohe Diskontierungsraten zukünftige Effekte heutiger Maßnahmen klein. Politisch könnten damit lästige zukünftige Probleme heutiger Entscheidungen marginalisiert und deren Lösung in die Zukunft transportiert werden. Dies muss im Rahmen der Auswahl der Höhe der Diskontierungsrate sowie des zugrunde liegenden Diskontierungsverfahrens unbedingt vermieden werden. ${ }^{11}$ Diese ineffiziente und ungerechte politische Verdrängung durch gezieltes „Klein-

9 Vgl. Bayer (2000), S. 54

10 Vgl. Stern (2006).

11 Ein Verfahren, das intergenerational zu mehr Neutralität führt, ist das Generation Adjusted Discounting (GAD), das für politische Langfristplanung eingesetzt werden könnte. Vgl. hierzu grundlegend Bayer (2004) sowie eine Anwendung im Bereich des Betriebs von Hausmülldeponien in Bayer/Méry (2009); hier berechnen die Autoren sog. „sustainability gaps“ als Diskontratendifferenzen zwischen der konventionellen Diskontierung und dem GAD. 
rechnen“ von Klimaschäden steht jedoch im Widerspruch zu parallel laufenden internationalen Bemühungen: Spätestens seit dem internationalen Bekenntnis zu einer nachhaltigen Entwicklung sollen heute lebende Generationen die Verantwortung für die jetzt und in der Vergangenheit emittierten Treibhausgase übernehmen und Reduktionsvereinbarungen umsetzen, um zukünftige Generationen in ihrer Lebensführung so wenig wie möglich zu beeinträchtigen. Wie das politisch gelingen kann, wollen wir im nachfolgenden Kapitel 3 analysieren.

\section{Handelbare Emissionsrechte zur Bekämpfung des Klimawandels}

Die internationale Klimapolitik ist verhältnismäßig jung. Ein erster wesentlicher Schritt in ihrer Entwicklung war die auf dem „Earth Summit“ in Rio de Janeiro 1992 verabschiedete Klimarahmenkonvention (United Nations Framework Convention on Climate Change, UNFCCC). Die sog. Annex-I-Länder, die Industrieländer und die im Transformationsprozess befindlichen Länder Osteuropas, haben sich dazu bekannt, ihre jährlichen Treibhausgasemissionen bis zum Jahr 2000 auf dem Stand von 1990 zu stabilisieren. Weil die in der Klimarahmenkonvention vereinbarte Konstanthaltung der Emissionen aus naturwissenschaftlicher Sicht nicht ausreicht, strebte man auf den seit 1995 jährlich abgehaltenen Vertragsstaatenkonferenzen an, weitere Reduktionen vertraglich zu fixieren. Auf der 3. Vertragsstaatenkonferenz in Kyoto gelang 1997 ein Durchbruch. Die Industrieländer verpflichteten sich im Kyoto-Protokoll zur Reduzierung der sechs wichtigsten Treibhausgase um mindestens 5,2 Prozent auf Basis des Jahres 1990 bis zu einer ersten Verpflichtungsperiode (2008 bis 2012). Es ermöglicht den einzelnen Staaten den Einsatz marktwirtschaftlicher Instrumente zur kostenminimalen Erreichung des nationalen Klimaschutzziels, also handelbare Emissionsrechte sowie zwei Unterarten (Joint Implementation und Clean Development Mechanism). Dabei wird jeweils die Eigenschaft der Treibhausgasemissionen als Globalschadstoff ausgenutzt: Jede vermiedene Emissionseinheit leistet den gleichen Beitrag zur Stabilisierung des Weltklimas. Ökonomische Überlegungen führen dazu, dass Emissionen dort vermieden werden, wo die niedrigsten Vermeidungskosten anfallen. Verfehlt z.B. ein Land sein vorgegebenes Ziel, so muss es Emissionsrechte von einem anderen Land kaufen, das sein Ziel übererfüllt. Die Einhaltung eines vorgegebenen Klimaschutzziels wird in diesem System zu den geringsten volkswirtschaftlichen Kosten erreicht. ${ }^{12}$

Das System der handelbaren Emissionsrechte (oder Umweltzertifikate) greift auf den preislichen Steuermechanismus zur Internalisierung externer Effekte zurück. ${ }^{13}$ Hier wird nicht - wie bei klassischen Auflagenlösungen - eine einzuhaltende Obergrenze politisch vorgegeben, die alle Normadressaten strikt einhalten müssen. Es wird auch kein politischer Preis je Schadstoffemissionseinheit (willkürlich) vorgegeben, an dem sich die Vermeidungsanstrengungen ausrichten. Bei handelbaren Emissionsrechten gibt die Politik stattdessen in Höhe der zulässigen 12 Vgl. Cansier/Bayer (2003), S. 150ff., Reichenbach/Requate (2008) und ausführ-
lich Schwarze (2000), S. 109-144.

13 Vgl. Cansier/Bayer (2003), S. 135-165.
Emissionen Berechtigungen aus. Die Emissionsrechte lauten auf eine bestimmte Schadstoffmenge in einer bestimmten Periode, beispielsweise auf das Recht zur Emission einer Tonne $\mathrm{CO}_{2}$ im Jahr 2009. Die Inverkehrbringung geschieht entweder durch Versteigerung oder durch freie Vergabe an die Altemittenten nach einem historischen Schlüssel oder anderen Kriterien. Insgesamt gilt also - wie bei Auflagenlösungen - eine politisch fixierte Emissionsobergrenze. Es wird allerdings nicht jedem Emittenten die gleiche Vermeidungsleistung oder die gleiche absolute Obergrenze abverlangt. Dies wäre aus ökonomischer Perspektive ineffizient, weil teure und billige Vermeider undifferenziert behandelt werden. Stattdessen können die Emittenten im Rahmen handelbarer Emissionsrechtesysteme die Rechte handeln: Emittenten mit niedrigen Vermeidungskosten sind in der Lage, zu den gleichen gesamten Vermeidungskosten mehr zu vermeiden als Emittenten mit hohen Vermeidungskosten. Handelbare Emissionsrechte ermöglichen somit bei Einhaltung des vorgegebenen Emissionsoberziels, dass Vermeidungsleistungen hauptsächlich von denjenigen Emittenten erbracht werden, die geringe Kosten für die Erbringung der politisch vorgeschriebenen Vermeidungsleistung haben. Dies ermöglicht den Mehrausstoß an Emissionen bei teureren Vermeidern, die dafür aber Emissionsrechte von den billigen Vermeidern kaufen müssen. In Abhängigkeit der Preise für Emissionsrechte ergibt sich dann ein Preis auf diesem Rechtemarkt, der sich durch Angebot von und Nachfrage nach Emissionsrechten automatisch einstellt. Hierin liegt der Unterschied zu den Umweltabgaben, bei denen sich durch eine exogene politische Preisvorgabe ein Umweltziel nur zufällig realisieren lässt. Umweltziele können im Rahmen handelbarer Emissionsrechte dagegen zielgenau und - bei funktionsfähigen Zertifikatemärkten (inkl. Sanktionierung bei Zielverfehlung) - mit den geringsten volkswirtschaftlichen Kosten realisiert werden: Jeder Emittent, der mehr als die ihm zugestandene Menge emittieren möchte, benötigt einen Verkäufer von Emissionsrechten, damit dies gelingen kann. Handelbare Emissionsrechtesysteme ermöglichen eine kostengünstige Anpassung aller im System befindlichen Emittenten bei der Aufteilung der tatsächlichen Vermeidungsmengen bei gleichzeitiger Sicherstellung einer politisch gewollten Höchstemissionsmenge.

Trotz der Klimarahmenkonvention und des Kyoto-Protokolls sind die tatsächlichen Emissionsentwicklungen aus Klimaschutzsicht eher ernüchternd. Seit 1990 steigen die weltweiten $\mathrm{CO}_{2}$-Emissionen stark an, insgesamt haben sich die energiebedingten $\mathrm{CO}_{2}$-Emissionen bis 2008 um 25 Prozent erhöht. ${ }^{14}$ Dieser Anstieg findet sowohl in den Entwicklungsländern als auch in den Industrieländern statt; Ausnahmen sind einzelne Länder, in denen die Emissionstätigkeit seit 1990 gesunken ist, etwa die Bundesrepublik und das Vereinigte Königreich sowie alle Transformationsländer des ehemaligen Osteuropa. ${ }^{15} \mathrm{Im}$ Vergleich zum Kyoto-Basisjahr von 1990 sind somit die Emissionen der nicht durch eine Emissionsobergrenze gebundenen Länder deutlich stärker angestiegen als die Emissionstätigkeit der durch nationale Obergrenzen gebundenen Länder gesenkt werden konnte. ${ }^{16}$ Bis 2030 wird prognostiziert, dass die Emissi-

14 Vgl. IEA (2008), S. 8

15 Vgl. etwa EEA (2008), S. 19

16 Vgl. IPCC (2007b), S. $261 \mathrm{f}$. 
onstätigkeit in den Annex-I-Ländern weiter ansteigt. Allerdings nehmen die treibhausrelevanten Emissionen in den Non-Annex-I-Ländern in den bekanntesten Prognosen deutlich schneller zu, so dass weltweit insgesamt von steigenden Treibhausgasemissionen ausgegangen werden muss. ${ }^{17}$

Skeptiker marktwirtschaftlicher Systeme zur Lösung von Umweltschutzproblemen führen die aus Klimaschutzsicht enttäuschende Emissionsentwicklung auch in Europa oftmals auf die Verwendung handelbarer Emissionsrechte zurück. Dieses Instrument - so dessen Kritiker - führe allenfalls dazu, dass eine Art Ablasshandel betrieben würde, ohne dass sich die tatsächlichen treibhausrelevanten Emissionen deutlich reduzieren würden. Diesem Einwand sollen zwei Argumente im Folgenden entgegengehalten werden: Zum einen das in der Klimarahmenkonvention im internationalen Rahmen gültige Prinzip der gemeinsamen aber differenzierten Verantwortung. Zum anderen wird anhand ausgewählter Ausgestaltungsfragen bei der Einführung des handelbaren Emissionsrechtesystems in der Bundesrepublik aufgezeigt, dass die durch die Politik festgelegten Rahmenbedingungen dazu führen, dass das Instrument der handelbaren Emissionsrechte völlig zu Unrecht für die allenfalls geringen Klimaschutzerfolge am Pranger steht.

Das erste Grundproblem bei der politischen Ausgestaltung des internationalen Klimaschutzes besteht im Grundsatz der gemeinsamen aber differenzierten Verantwortung des Artikels 3 der Klimarahmenkonvention der Vereinten Nationen. ${ }^{18}$ Dieser Grundsatz besagt, dass es sich beim Klimawandel um ein internationales Problem handelt, die einzelnen Länder der Staatengemeinschaft aber bislang in unterschiedlichem Ausmaß dazu beigetragen haben. Insbesondere die Entwicklungsländer besitzen auf Grund von Gerechtigkeitserwägungen deshalb ein „Recht auf nachholende Entwicklung“, was bei den Klimaschutzanstrengungen zu berücksichtigen ist. Aus diesem Grunde differenziert die Klimarahmenkonvention zwischen den Annex-I und Non-Annex-I-Ländern. ${ }^{19} \mathrm{Zu}$ den Non-Annex-I-Ländern („Entwicklungsländer“) zählen auch China, Indien, Brasilien und weitere „Schwellenländer”. Sowohl im Rahmen der Klimarahmenkonvention als auch im 1997 folgenden Kyoto-Protokoll ${ }^{20}$ müssen die Annex-I-Staaten ihre treibhausrelevanten Emissionen reduzieren oder zumindest begrenzen; insgesamt sieht das Kyoto-Protokoll eine durchschnittliche Reduzierung der treibhausrelevanten Emissionen um 5,2 Prozent bis zur ersten Verpflichtungsperiode vor, die von 2008 bis 2012 andauert. Basisjahr für die Reduktions- bzw. Begrenzungszusagen ist in der Regel das Jahr 1990. Für die Entwicklungsländer besteht dagegen das Recht, Emissionen ohne jegliche Begrenzung ausstoßen zu dürfen. Insofern besteht im Kyoto-Protokoll hinsichtlich der Erreichung einer weltweiten Reduktion der gesamten Treibhausgasemissionstätigkeit ein politisches Steuerungsdefizit: Nur wenn die Mehremissionen in den Entwicklungsländern in der Summe unter der Reduktion der Industrie- und Transformationsländer bliebe, könnte eine weltweite Reduktion der Treibhausgasemissionen realisiert

17 Vgl. IPCC (2007b), S. 262f.

18 Vgl. UNFCCC (1992), Artikel 3 und 4, S. 5 ff.

19 Vgl. UNFCCC (1992), Anlage 1, S. $24 \mathrm{f}$.

20 Vgl. UNFCCC (1997), dort wird von Annex-B und Non-Annex-B-Ländern gesprochen; faktisch sind Annex-I- und Annex-B-Staaten weitgehend identisch. werden. Die tatsächlichen weltweiten Emissionsverläufe zeigen jedoch, dass diese Hoffnung aller am Klimaschutzprozess beteiligten Staaten sich bislang nicht erfüllt hat. Ursächlich hierfür sind hauptsächlich die sehr hohen Emissionszuwächse v.a. in China und Indien; erwähnt werden muss aber auch die teilweise Nicht-Einhaltung zugesagter Vermeidungs- bzw. Begrenzungsleistungen einzelner Industriestaaten (etwa USA, Spanien oder Portugal ${ }^{21}$ ).

Zur politischen Steuerung der Treibhausgasemissionen sehen sowohl die Klimarahmenkonvention (zumindest indirekt über die Forderung der kostengünstigen Erreichung politisch fixierter Emissionsziele, Artikel 3, Abs. 3) als auch das KyotoProtokoll (hier direkt in Artikel 17) den Einsatz des Instruments handelbarer Emissionsrechte vor. Dies liegt vor allem daran, dass handelbare Emissionsrechtesysteme grundsätzlich die Eigenschaft aufweisen, den angestrebten Klimaschutz zu den geringst möglichen volkswirtschaftlichen Kosten zu erlangen. Inwieweit die grundsätzliche Eigenschaft der Kosteneffizienz allerdings erreicht werden kann, hängt wesentlich von der politischen Ausgestaltung des Instruments ab. ${ }^{22}$ Anders ausgedrückt: Nur wenn bestimmte Mindestanforderungen bei der Ausgestaltung eines handelbaren Emissionsrechtesystems existieren, kann das Instrument seine volle Stärke auch entfalten.

Das Kyoto-Protokoll gibt für die grundsätzlichen Ausgestaltungsfragen keinen Rahmen vor. Sämtliche Ausgestaltungsfragen werden damit der politischen Souveränität der Unterzeichnerstaaten überlassen. In Europa hat die EU-Kommission Rahmenbestimmungen für die Einführung eines handelbaren Emissionsrechtesystems in der EU vorgegeben, die zum 1.1.2005 in Kraft getreten sind. Seit dem 1.1.2008 ist auch in der Bundesrepublik ein handelbares Emissionsrechtesystem etabliert worden, das die Reduktion treibhausrelevanter Emissionen in einzelnen volkswirtschaftlichen Sektoren bewerkstelligen soll. ${ }^{23}$ Dieses System bewegt sich erstens im Rahmen, den die EU-Kommission der Bundesrepublik zum Einsatz dieses Instruments vorgegeben hat. Zweitens muss berücksichtigt werden, dass in der Bundesrepublik bereits vor Einführung des handelbaren Emissionsrechtesystems Instrumente implementiert worden sind, mit denen Klimaschutzziele erreicht werden sollten. Zu erwähnen sind hier vor allem eine Fülle von Auflagen, ${ }^{24}$ die freiwillige Selbstverpflichtungserklärung der deutschen Wirtschaft zum Klimaschutz ${ }^{25}$ sowie die „Ökosteuer“26. Da zu keinem Zeitpunkt die Abschaffung der bestehenden Instrumentenvielfalt in der Bundesrepublik bei gleichzeitiger Einführung eines handelbaren Emissionsrechtesystems zum Klimaschutz politisch diskutiert wurde, ist es klar, dass die Ausgestaltung des handelbaren Emissionsrechtesystems unter Berücksichtigung der bestehenden Instrumente stattfand. Insofern überrascht es nicht, dass die theoretischen Anforderungen an ein effizientes

21 Vgl. UNFCCC (2009).

22 Vgl. Bayer/Cansier (1999), S. 263, für die Auflistung der zentralen Ausgestaltungsfragen kosteneffizienter handelbarer Emissionsrechtesysteme.

23 Vgl. BMU (2007) für die Grundlagen dieses Systems.

24 Gesetzliche Grundlage eines Großteils der Auflagen sind das Bundesimmissionsschutzgesetz (BImSchG) sowie die sich daran anschließenden Bundesimmissionschutzverordnungen (BImSchV) einschließlich einiger technischer Anleitungen (TA). Auflagen sind das „klassische“ Instrument der Umweltpolitik in der Bundesrepublik und dominieren bislang die umweltpolitische Praxis.

25 Vgl. $B M U(2000)$

26 Vgl. $B M U$ (2008). 
handelbares Emissionsrechtesystem nur in rudimentären Zügen Berücksichtigung finden konnten und die praktische Ausgestaltung des Instruments innerhalb enger politischer Zwänge stattfand. Beispielhaft sei die kostenlose Vergabe der Rechte erwähnt, obgleich die Europäische Direktive eine Möglichkeit zur Versteigerung bis zu zehn Prozent vorsah. ${ }^{27}$ Zudem hat die Politik festgelegt, dass nur Anlagen zertifikatepflichtig sind, die eine gewisse Mindestgröße und damit einen Mindestausstoß aufweisen. Damit begibt sich der Gesetzgeber der Möglichkeit, dass ein Ausgleich von Vermeidungskosten über alle Emittenten hinweg stattfinden kann. Somit ist das Instrument aus ökonomischer Perspektive nur in der Lage, suboptimale Ergebnisse zu produzieren. Zudem werden die beiden Sektoren „private Haushalte“ sowie „Verkehr“ grundsätzlich von der Emissionsrechtepflichtigkeit ausgenommen. Auch durch diese Entscheidung wird der Kreis derjenigen aus politischen Erwägungen verkleinert, die am handelbaren Emissionsrechtemarkt hätten teilnehmen können. Darüber hinaus beinhaltet das handelbare Emissionsrechtesystem in der Bundesrepublik noch eine Fülle an Ausnahmen, die die effiziente Funktionsfähigkeit des Systems bereits bei dessen Einführung massiv in Frage stellen. Deshalb sei an dieser Stelle nochmals angemerkt: Die bislang nicht guten Erfahrungen mit dem Instrument der handelbaren Emissionsrechte in der Bundesrepublik liegen nicht am Instrument als solchem, sondern vielmehr daran, dass bei der politischen Ausgestaltung wenig Augenmerk auf die Erreichung der ökonomischen Effizienz (kostenminimale Erreichung des vorgegebenen politischen Ziels) gelegt wurde. Insofern muss aus umweltökonomischer Sicht ein Politik- und nicht ein Instrumentaldefizit oder gar -versagen festgehalten werden.

\section{Fazit}

Klimaschutz ist eine Langfristaufgabe, die häufig im politischen Kontext mit Kurzzeiterfordernissen im Konflikt steht. Dies verlangt nach einer Lösung, die beide Aspekte zugleich berücksichtigt. Am einfachsten ließe sich Klimaschutz dadurch bewerkstelligen, dass ein alle Emittenten einbeziehendes handelbares Emissionsrechtesystem etabliert würde. Dies impliziert jedoch, dass bestehende Instrumente möglicherweise ausgesetzt werden müssten, und mithin - etwa bei Verzicht auf die Ökosteuer - erhebliche Finanzierungslücken im gesetzlichen Rentenversicherungssystem entstehen würden. Diese und weitere Rückwirkungen singulärer politischer Entscheidungen erschweren einen umfassenden Klimaschutz erheblich. Für die politische Ausgestaltung des handelbaren Emissionsrechtesystems müsste darauf geachtet werden, dass so wenig Ausnahmentatbestän-

\footnotetext{
27 Die Vergabemodalität beeinflusst die Wirkungsweise von handelbaren Emissionsrechten an sich nicht. Auch dass die Verbraucher für den Klimaschutz bezahlen, ist sowohl bei der kostenlosen Vergabe wie bei einer Versteigerung identisch. Bei der Versteigerung von Zertifikaten profitiert der Staat als Verkäufer der Rechte und erhöht seine Einnahmen. Auf diese verzichtet er bewusst bei der kostenfreien Vergabe und ermöglicht zusätzliche Einnahmen auf Seiten der Zertifikatepflichtigen. Das Aneignen der sogenannten „Windfall Profits“ wurde v.a. den großen Energieversorgern vorgeworfen. Allerdings wurde deren Verhalten bereits vor Inkrafttreten des Treibhausgasemissionshandelsgesetzes (TEHG) prognostiziert - bereits ex-ante absehbar verhielten sich diese Akteure ökonomisch völlig rational. Der eigentliche Adressat von Kritik sollte die Politik sein, die für diese Art der Ausgestaltung Verantwortung trägt. Vgl. hierzu etwa Erdmann (2005).
}

de wie möglich vereinbart werden. In der Realität wären durch deren Reduktion bzw. Abschaffung gegenüber der jetzigen Situation deutliche Effizienzverbesserungen möglich.

Voraussetzung für die Implementierung eines handelbaren Emissionsrechtesystems stellt allerdings die politische Formulierung eines verbindlichen möglichst internationalen Klimaschutzzieles dar, das - wie in der Klimarahmenkonvention gefordert - gefährliche Auswirkungen des Klimawandels minimiert und Irreversibilitäten vermeidet. ${ }^{28}$ Damit müsste ein Verfahren für die politische Langfristplanung eingesetzt werden, das sowohl die heutigen politischen Dringlichkeiten als auch die zukünftigen besser miteinander in Beziehung setzt. Trotz der individuellen Kurzsichtigkeit, die allen Menschen innewohnt und im politischen Kalkül durch (aus Klimaschutzsicht) sehr kurze Legislaturperioden besonders virulent ist, müsste dieses Planungsverfahren - wie etwa das skizzierte Generation Adjusted Discounting (GAD) - zukünftige Entwicklungen stärker in den politischen Fokus nehmen, um nicht durch einfache Rechenoperationen zukünftigen Generationen einen Berg von ungelösten Problemen zu hinterlassen, deren Lösung bei relativ zeitnahen Aktivitäten in der Summe weltweit sowie jeweils national möglicherweise deutlich geringere Kosten verursacht hätte.

\section{Literatur}

Bayer, Stefan (2008): Wirtschaft, Umwelt, Ressourcen und Sicherheit, in: Böckenförde, Stephan und Sven Bernhard Gareis (Hrsg.): Deutsche Sicherheitspolitik in einer Welt im Wandel. Herausforderungen, Akteure und Strategien, Leverkusen: Verlag Barbara Budrich, S. 173-208.

Bayer, Stefan (2004): Nachhaltigkeitskonforme Diskontierung - Das Generation Adjusted Discounting, DIW-Vierteljahreshefte zur Wirtschaftsforschung, Schwerpunktheft „Messung von Nachhaltigkeit“, Vol. 73, Heft 1, S. 142-157.

Bayer, Stefan (2000): Intergenerationelle Diskontierung am Beispiel des Klimaschutzes, Marburg: Metropolis.

Bayer, Stefan und Dieter Cansier (1999): Kyoto-Mechanismen und globaler Klimaschutz. Die Rolle handelbarer Emissionsrechte, Hamburger Jahrbuch für Wirtschafts- und Gesellschaftspolitik, Band 44, S. 255-273.

Bayer, Stefan und Jacques Méry (2009): Sustainability gaps in municipal solid waste management: a case study for landfills, Environment, Development and Sustainability, Vol. 11, No. 1, S. 43-69.

Benz, Eva und Bodo Sturm (2008): Weichenstellung für den europäischen Emissionshandel, Wirtschaftsdienst, 88. Jahrgang, Heft 12, S. 810-813.

Bundesministerium für Umwelt, Naturschutz und Reaktorsicherheit (BMU) (2008): Links zum Thema Ökologische Steuerreform, http://www.bmu.de/wirtschaft_und_umwelt/oekologische_finanzreform/ doc/2508.php, zuletzt geladen am 5. Februar 2009.

28 Vgl. UNFCCC (1992), Art. 3. 
Bundesministerium für Umwelt, Naturschutz und Reaktorsicherheit (BMU) (2007): Gesetz über den Handel mit Berechtigungen zur Emission von Treibhausgasen (Treibhausgas-Emissionshandelsgesetz -TEHG), Berlin.

Bundesministerium für Umwelt, Naturschutz und Reaktorsicherheit (BMU) (2000): Selbstverpflichtungserklärung der Deutschen Wirtschaft, Berlin.

Cansier, Dieter und Stefan Bayer (2003): Einführung in die Finanzwissenschaft. Grundfunktionen des Fiskus, München und Wien: Oldenbourg.

Erdmann, Georg (2005): Klimaschutz oder Interessenpolitik: Über einige ungewollte Resultate ökonomischer Politikberatung, Perspektiven der Wirtschaftspolitik, Band 6, Heft 3, S. 347-367.

Europäische Kommission (2003): Richtlinie 2003/87/EG des Europäischen Parlaments und des Rates vom 13. Oktober 2003 über ein System für den Handel mit Treibhausgasemissionszertifikaten in der Gemeinschaft und zur Änderung der Richtlinie 96/61/EG des Rates, Brüssel.

European Energy Agency (EEA) (2008): Greenhouse gas emission trends and projections in Europe 2008, Tracking progress towards Kyoto target, EEA Report No 5/2008, Copenhagen.

International Energy Agency (IEA) (2008): World Energy Outlook 2008. Presentation to the Press, http://www.iea.org/Textbase/ speech/2008/Birol_WEO2008_PressConf.pdf, zuletzt geladen am 8. Februar 2009.

Intergovernmental Panel on Climate Change (IPCCa) (2007): Climate Change 2007. The Physical Science Basis, Contribution of Working Group I to the Fourth Assessment Report of the IPCC, Cambridge.

Intergovernmental Panel on Climate Change (IPCCb) (2007): Climate Change 2007. Mitigation of Climate Change, Working Group III contribution to the Fourth Assessment Report of the IPCC, Cambridge.

Lomborg, Björn (2007): Cool it. The skeptical environmentalist's Guide to Global Warming, New York: Knopf.

Nordhaus, William D. (1994): Managing the Global Commons. The Economics of Climate Change, Cambridge, Mass. - London: MIT-Press.

Reichenbach, Johanna und Till Requate (2008): Umweltpolitische Instrumente in Theorie und Praxis, in: Varwick, Johannes (Hrsg.): Globale Umweltpolitik. Eine Einführung, Schwalbach, Ts.: Wochenschau-Verlag, S. 94-122.

Schwarze, Reimund (2000): Internationale Klimapolitik, Marburg: Metropolis.

Stern, Nicholas (2006): The Economics of Climate Change, http://www.hm-treasury.gov.uk/ stern_review_report.htm, zuletzt geladen am 30. Januar 2009.

UNFCCC (2009): GHG-Data from UNFCCC, http://unfccc.int/ ghg_data/ghg_data_unfccc/items/4146.php, zuletzt geladen am 9. Februar 2009.
UNFCCC (1997): Protokoll von Kyoto zum Rahmenübereinkommen der Vereinten Nationen über Klimaänderungen (Kyoto-Protokoll), Kyoto.

UNFCCC (1992): Rahmenübereinkommen der Vereinten Nationen über Klimaänderungen (Klimarahmenkonvention), New York.

Wissenschaftlicher Beirat der Bundesregierung Globale Umweltveränderungen (2007): Welt im Wandel - Sicherheitsrisiko Klimawandel, Berlin und Heidelberg: Springer.

World Commission on Environment and Development (1987): Our common Future, Oxford - New York: Oxford University Press (Brundtland-Bericht).

Ziesing, Hans-Joachim (2006): Trotz Klimaschutzabkommen: Weltweit steigende $\mathrm{CO}_{2}$-Emissionen, DIW-Wochenbereicht, 73. Jahrgang, Nr. 35/2006, S. 485-499.

\section{Neu im Programm}

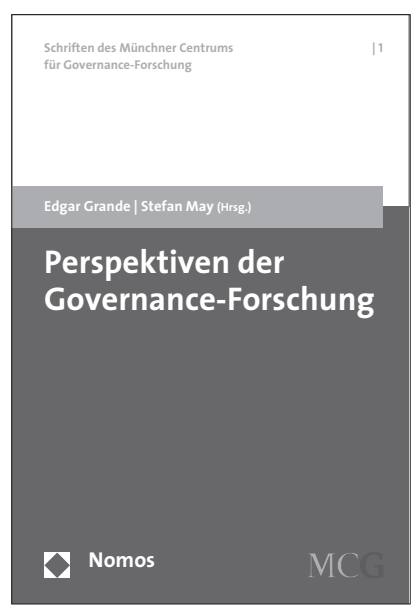

\section{Perspektiven der Governance-Forschung}

Herausgegeben von Edgar Grande und Stefan May 2009, 91 S., brosch., 14,- $€$,

ISBN 978-3-8329-4287-8

(Schriften des Münchner Centrums

für Governance-Forschung, Bd. 1)

Ziel des Bandes ist, Stand und Ausblick der GovernanceForschung als auch das Forschungsprogramm des Münchner Centrums für Governance-Forschung interdisziplinär zu diskutieren. Der Band eröffnet zugleich eine Schriftenreihe, in der in den kommenden Jahren die Ergebnisse jener Forschungsarbeit vorgestellt werden sollen.

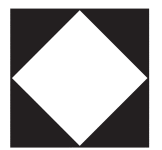

\section{Nomos}

Bitte bestellen Sie im Buchhandel oder versandkostenfrei unter www.nomos-shop.de 